\title{
MODELING DEBRIS FLOW: ON THE INFLUENCE OF PORE PRESSURE EVOLUTION AND HYPOPLASTICITY
}

\author{
JULIAN HEß \& YONGQI WANG \\ Institute of Fluid Dynamics, Technische Universität Darmstadt, Germany.
}

\begin{abstract}
For granular debris flows, two characteristics play a crucial role in their dynamic behavior: the porepressure feedback, which reduces the intergranular friction and, therefore, enhances the mobility of the whole mixture, and the non-linear deformational behavior that stems from the internal contact stress between grains. In a previous work, the entropy principle based on the formulation of Müller and Liu was exploited in order to find restrictions on the constitutive equations of a general grain-fluid multiphase mixture, including two additional internal variables. In this report, a thermodynamically consistent model for debris flows is depth-integrated and employed for numerical simulation.

Including extra pore-pressure and hypoplastic stress, internal variables that are, respectively, described by a pressure diffusion equation and a transport equation related to the hypoplastic material, are considered. Comparison of the obtained results with those from classical debris flow models shows that the proposed thermodynamic model provides a phenomenological insight into the influence of the pore-pressure feedback and intergranular friction in the flow dynamics.

To better understand the significance of the pore-pressure feedback and the intergranular friction, a simple grain-fluid material sliding on a slope with runout is numerically investigated by using depthintegrated model equations. A non-oscillatory, shock-capturing central-upwind scheme with the total variation diminishing property is applied for this purpose. Numerical results indicate the significant importance of the pore-pressure feedback and the hypoplastic behavior on determining the flow dynamics of debris flows.
\end{abstract}

Keywords: debris flow, extra pore fluid pressure experiments, granular-fluid mixture, hypoplasticity, Müller-Liu entropy principle

\section{INTRODUCTION}

Debris flows are large mixtures of granular materials and fluids that can, due to their rapid mass movement driven by gravity, travel long distances in mountainous regions. Since debris flows occur both in nature and technical processes, they represent an important case of granular-fluid flows. Furthermore, these kinds of flows are dangerous for people and structures, causing severe casualties and destruction due to the immense masses they carry. Prevention measures require an advanced understanding of the flow dynamics, which are hard to examine due to the complex physical mechanisms, arising from the interaction of multiple phases. Unlike mud flows, which tend to be rather fluid-like, or dry rock avalanches, being hindered in their dynamics by the relatively large frictional forces, in debris flow, the concurrence of a lubricating fluid phase and a ponderous solid phase enhance the flow dynamics. The following work tries to gain insight into these dynamics and the underlying physical mechanisms of their driving forces.

Since modeling faces the problem of depicting complex interactions by fairly simple equations, one needs to disregard insignificant mechanisms. Therefore, proposing a new model, careful investigation of the influence of included physical mechanisms is necessary for further and deeper understanding of the internal behavior as well as for the conceivable exclusion of particular mechanisms. On account of this, in our model, the incorporation of additional variables, an internal frictional stress and the extra pore pressure goes back to previous findings in experiments and resulting attempts to model the behavior of granular materials and debris flow. 
Hypoplastic models for the deformational behavior of granular materials, describing the different paths of loading and unloading, have been developed in a series of publications by Refs. [1-3]. Hypoplasticity itself is a rather simple model, developed in soil mechanics to facilitate previous, more sophisticated concepts, while retaining the core ability of accurately predicting the deformation of granular material as both anelastic and non-linear. Hypoplasticity predicts a certain relation between the strain rate, i.e. the deformation due to shear, and an internal contact-stress, modeled as a symmetric second-order tensorial variable.

In addition, another well-known concept from soil mechanics is considered due to its explanatory power in terms of the increased mobility of debris flow. According to Terzaghi's concept of effective stress in porous media, see Refs. [4, 5], a dynamic extra pore-fluid pressure is considered to mediate emerging load on the solid structure to the fluid. This line of tradition in debris flow modeling goes mainly back to the works of Iverson et al., see Refs. [6-9].

Because of the physical mechanisms apparent in debris flow, both hypoplastic behavior and the evolution of a dynamic extra pressure were incorporated in the model. The development of a thermodynamically consistent model according to the entropy principle in its formulation by Ref. [10], see also Refs. [11, 12], has been carried out in a previous publication, see Ref. [13]. Firstly incorporating these two quantities in the modeling process, we proceed here with the derivation of an applicable model for debris flow. With this, the influence of these additional quantities on the material equations is identified and, therefore, their interaction and the general flow dynamics can be further depicted. It is shown that especially during the onset and the settling of flows, the extra pore-fluid pressure and the internal friction capture significant aspects of debris flow behavior that have not been considered yet.

In this work, the set of depth-integrated equations is utilized for the numerical simulation of a simple debris flow with an Central upwind scheme and the influence of the distinct terms is examined.

\section{EQUATIONS}

In previous work, see Ref. [13], we developed restrictions posed by the second law of thermodynamics on the constitutive relations, for a system of equations within the framework of mixture theory, based first and foremost on the work of Ref. [14].

The point of origin of the modeling process is the definition of a set of equations that describe the physical mechanisms of the system via a range of fields. The fields described by these balance equations are the true density $\rho^{\alpha}$ and the volume fraction $v^{\alpha}$ of the constituents $\alpha$, the partial velocity $v_{i}^{\alpha}$, the inner stress $Z_{i j}^{\alpha}$ and the partial pressure $\varpi^{\alpha}$. Within the momentum balance, the stress tensor $T_{i j}^{a}$ describes the mechanisms of load transmission within the material, as does the momentum interaction term $m_{i}^{a}$ between the different phases. By this means, thermodynamically consistent constitutive equations were developed most notably for the stress tensor and the momentum interaction term with regard to the chosen set of balance equations. In consequence, the property of consistency here also means that the structure of the constitutive equations reflects the initial system of equations, for which the constitutive equations themselves are developed. Here, we tie in with closure suggestions made by Refs. [15] and [16], linking the results of the Müller-Liu derivation to the pore-fluid pressure. After this step, a closed set of equations has been developed. Also note that, although we employed the energy balance and the mass balance for the exploitation of the entropy principle, for this work, we assume the partial density and the mixture temperature to be constant.

In Ref. [13], it follows for the solid and fluid stress tensor, that 


$$
\begin{aligned}
& T_{i j}^{s}=-\left(\varpi_{h}^{f}-C_{e}^{f} \varpi_{e}^{f}+\varpi_{v}^{s}-\left(\rho^{s}-\rho^{f}\right) \delta_{Z} Z_{k k}^{s}\right) v^{s} \delta_{i j}+\rho \delta_{Z} Z_{i j}^{s} \\
& T_{i j}^{s}=-\left(\varpi_{h}^{f}-C_{e}^{f} \varpi_{e}^{f}\right) v^{f} \delta_{i j}+a_{1}^{f} D_{k k}^{f} \delta_{i j}+a_{2}^{f} D_{i j}^{f}
\end{aligned}
$$

with an configuration pressure $\varpi_{v}^{s}=\left(\frac{\rho^{s}}{\rho^{f}}-1\right) \varpi_{h}^{f}$, the hydrostatic fluid pressure $\varpi_{h}^{f}$ and an extra pore-fluid pressure $\varpi_{e}^{f}$, multiplied by a coefficient $C_{e}^{f}$. In the solid stress tensor, the intergranular contact stress is considered with $\delta_{Z} Z_{i j}^{s}$. Quantities without superscript $s, f$ are mixture quantities, like the mixture density $\rho$. The spin tensor $W_{i j}^{a}=\frac{1}{2}\left(\partial_{x_{j}} v_{i}^{a}-\partial_{x_{i}} v_{j}^{a}\right)$ and the strain tensor $D_{i j}^{a}=\frac{1}{2}\left(\partial_{x_{j}} v_{i}^{a}+\partial_{x_{i}} v_{j}^{a}\right)$ result from the gradients of the partial velocities.

Furthermore, the momentum interaction term in $x$-direction is given as

$$
\frac{m_{x}^{s}}{\rho^{a}}=c_{D}^{s}\left(v_{x}^{f}-v_{x}^{s}\right)+\varepsilon \frac{\partial v^{s}}{\partial x}\left(\varpi_{h}^{f}-\frac{v^{f} \rho^{f}}{\rho} \varpi_{v}^{s}\right)=-m_{x}^{f}
$$

with a drag coefficient

$$
c_{D}^{s}=v^{s} v^{f} g
$$

where $g$ is the acceleration due to gravity. At this point, we refrain from the implementation of a far more complex drag term, as postulated by Ref. [17], in order to firstly investigate the newly introduced quantities. The stress tensor exhibits the classical splitting with a pressure-like spherical part $\varpi^{a} v^{\alpha}$ and a deviatoric extra stress $T_{i j}^{e, a}$. While the momentum interaction term also possesses classical structure, including a drag term and a buoyant term, it is worth noting here that an additional term in conjunction with the configurational pressure $\varpi_{v}^{s}$ exists. This term is a direct result from the thermodynamically consistent derivation, usually not included in postulated buoyant terms of debris flow models. The structure of the terms (1)-(2) is a result from the thermodynamically consistent derivation and distinct closure assumptions.

We now proceed towards an application for two phase debris flow, by taking into account a slope without complex terrain or bed curvature. After the scaling analysis, a set of depth-integrated equations is derived by employing the shallow layer assumption. This reduction from $2 \mathrm{D}$ to $1 \mathrm{D}$ by vertical integration is a preliminary work to efficient numerical simulation in the following. Unlike in the model of Ref. [18] and the many successive works, this reduction is not strictly necessary for closure, since a constitutive model for the stress tensors of the solid and the fluid has been developed. Please note that, for the sake of brevity, we do not give any details on the derivation of the balance of mass and momentum. Furthermore, we omit overbar symbols, accounting for depth-integrated quantities. For the mass balance of the solid and fluid phases, it follows that

$$
\begin{aligned}
& \frac{\partial}{\partial t}\left(h v^{\mathrm{s}}\right)+\frac{\partial}{\partial x}\left(h v^{s} v_{x}^{s}\right)=0, \\
& \frac{\partial}{\partial t}\left(h v^{f}\right)+\frac{\partial}{\partial x}\left(h v^{f} v_{x}^{f}\right)=0 .
\end{aligned}
$$

The solid momentum balance in downslope direction is 


$$
\begin{aligned}
& \frac{\partial}{\partial t}\left(h v^{s} v_{x}^{s}\right)+\frac{\partial}{\partial x}\left(h v^{s} v_{x}^{s} v_{x}^{s}\right) \\
& =h v^{s} \sin (\vartheta)-\varepsilon \frac{\partial}{\partial x}\left(\left(1-\frac{\rho^{f}}{\rho^{s}}\right) v^{s} \cos (\vartheta) \frac{h^{2}}{2}-\operatorname{Eu} \frac{v_{e}^{s} \varpi_{e}^{f} h}{\rho^{s}}\right)-\frac{\varepsilon}{\rho^{s}} N_{Z} \frac{\partial \rho h Z_{z z}^{s}}{\partial x} \\
& -\left(\left(1-\frac{\rho^{f}}{\rho^{s}}\right) v^{s} h \cos (\vartheta)-\operatorname{Eu} \frac{\varpi_{e}^{f}}{\rho^{s}}\right) \operatorname{sgn}\left(v_{x}^{s}\right) \tan \left(\phi_{\mathrm{bed}}\right) \\
& +\frac{c_{D}^{s} h}{\rho^{s}}\left(v_{x}^{f}-v_{x}^{s}\right)-\varepsilon \frac{\rho^{f}}{\rho^{s}} v^{s} \cos (\vartheta) \mathrm{h} \frac{\partial h}{\partial x}+\varepsilon \frac{\rho^{f}}{\rho^{s}} \frac{v^{f}\left(\rho^{s}-\rho^{f}\right)}{\rho} \frac{h^{2}}{2} \cos (\vartheta) \frac{\partial v^{s}}{\partial x}
\end{aligned}
$$

and for the fluid

$$
\begin{aligned}
& \frac{\partial}{\partial t}\left(h v^{f} v_{x}^{f}\right)+\frac{\partial}{\partial x}\left(h v^{f} v_{x}^{f} v_{x}^{f}\right) \\
& =h v^{f} \sin (\vartheta)-\varepsilon \frac{\partial}{\partial x}\left(v^{f} \cos (\vartheta) \frac{h^{2}}{2}+\mathrm{Eu} \frac{v^{f} \bar{\varpi}_{e}^{f} h}{\rho^{f}}\right) \\
& +\frac{\varepsilon}{\rho^{f}} \mathrm{Ga}^{-\frac{1}{2}}\left(a_{1}^{f}+a_{2}^{f}\right) \frac{\partial}{\partial x}\left(h \frac{\partial v_{x}^{f}}{\partial x}\right)-\frac{a_{f} v^{f}}{\rho^{f}} v_{x}^{f} \mid b-\frac{c_{D}^{s} h}{\rho^{f}}\left(v_{x}^{f}-v_{x}^{s}\right) \\
& -\varepsilon \cos (\vartheta) \frac{h^{2}}{2} \frac{\partial v^{s}}{\partial x}-\varepsilon \frac{v^{f}\left(\rho^{\mathrm{s}}-\rho^{f}\right)}{\rho} \frac{h^{2}}{2} \cos (\vartheta) \frac{\partial v^{s}}{\partial x}
\end{aligned}
$$

For the development of the pressure, the depth-integrated evolution equation states

$$
\begin{aligned}
& \frac{\partial}{\partial t}\left(h \varpi_{e}^{f}\right)+\frac{\partial}{\partial x}\left(h \varpi^{f} v_{x}^{f}\right)-\left[\kappa_{\varpi}^{f} \frac{\partial \varpi_{e}^{f}}{\partial z}\right]_{b}^{s}= \\
& \frac{\partial}{\partial t}\left(h\left(\sigma-\varpi_{h}^{f}\right)\right)+\frac{\partial}{\partial t}\left(h\left(\sigma-\varpi_{h}^{f}\right) v_{x}^{f}\right)+\varpi_{e}^{f}\left(\frac{\partial h}{\partial t}+\frac{\partial v_{x}^{f} h}{\partial x}\right)-h \dot{\gamma} \tan (\psi)
\end{aligned}
$$

For this, it is assumed that the diffusion coefficient $\kappa_{\sigma}^{f}$ is not changing with depth. Furthermore, stating that the volume fraction is approximately constant with depth, $\partial_{z} v^{f} \approx 0$ is applied, following from the depth-integrated versions of the mass balance (4). To close this eq. (7), we consider the following relations

1. $\left[\kappa_{\varpi}^{f} \frac{\partial \varpi_{e}^{f}}{\partial z}\right]_{b}^{s}=2 \frac{\kappa_{\varpi}^{f}}{h} \varpi_{e}^{f}=2 \frac{\kappa_{m}}{h \mu^{s} \alpha_{D}} \varpi_{e}^{f}$,

2. $\frac{\partial\left(\sigma-\varpi_{h}^{f}\right)}{\partial t} h+\frac{\partial\left(\sigma-\varpi_{h}^{f}\right)}{\partial x} h v_{x}^{f}=\frac{1}{2} g h\left(\rho-\rho^{f}\right) \cos (\vartheta)\left(\frac{\partial h v^{s}\left(v_{x}^{s}-v_{x}^{f}\right)}{\partial x}-h \frac{\partial v_{x}^{f}}{\partial x}\right)$

3. $\varpi_{e}^{f}\left(\frac{\partial h}{\partial t}+\frac{\partial h v_{x}^{f}}{\partial x}\right)=\varpi_{e}^{f} \frac{\partial h v^{s}\left(v_{x}^{s}-v_{x}^{f}\right)}{\partial x}$,

4. $h \dot{\gamma} \tan (\psi)=2 \sqrt{\left(v_{x}^{s}\right)^{2}}\left(\kappa_{1} v^{s}-\kappa_{2} \frac{v_{C}^{s}}{1+\sqrt{N_{\psi}}}\right)$ 
where we have

$$
N_{\psi}=\frac{\mu^{s} \dot{\gamma}}{\rho^{s}\left(\dot{\gamma} \delta_{p}\right)^{2}+\sigma_{e}}, \dot{\gamma}=\frac{2 \sqrt{\left(v_{x}^{s}\right)^{2}}}{h}, \sigma_{e}=\left(\rho-\rho^{f}\right) g \cos (\vartheta) h,
$$

and two coefficients $\kappa_{\varpi 1}, \kappa_{\varpi 2}$. The pore-pressure diffusivity $\kappa_{\varpi}^{f}=\kappa_{m} /\left(\alpha_{D} \mu^{f}\right)$ is composed of the debris compressibility $a_{D}=\frac{a_{D}}{v^{s}\left(\sigma_{0}+\sigma_{e}\right)}$ and the hydraulic permeability $\kappa_{m}=\kappa_{0} \exp \left(\frac{0.6-v^{S}}{0.04}\right)$, while $\Delta_{i}^{f,}=\partial_{x_{i}} \kappa^{f}$

For the $x z$-balance equation of the hypoplastic stress, it follows that

$$
\begin{aligned}
& \varepsilon \frac{\partial h Z_{x z}^{s}}{\partial t}+\varepsilon \frac{\partial h v_{x}^{s} Z_{x z}^{s}}{\partial x}+\left(Z_{z z}^{s} h-Z_{x x}^{s} h\right)\left[v_{x}^{s}\right]_{b}^{s} \\
& -\varepsilon\left(1+\frac{v^{s}\left(\rho^{s}-\rho^{f}\right)}{\rho}\right) Z_{x z}^{s}\left(\frac{\partial h}{\partial t}+\frac{\partial v_{x}^{s} h}{\partial x}\right)-\varepsilon \Phi_{x z}^{s} h,
\end{aligned}
$$

with a depth-integrated, non-dimensional source term

$$
\begin{aligned}
& \Phi_{x z}^{s} h=f_{s}\left(a_{z}^{s}\left[v_{x}^{s}\right]_{b}^{s}+\varepsilon \frac{Z_{x z}^{s} Z_{x z}^{s} h\left(\frac{\partial v_{x}^{s} h}{\partial x}-\left[v_{x}^{s} \frac{\partial h}{\partial h}\right]\right)}{\left(Z_{x x}^{s}+Z_{z z}^{s}\right)^{2}}+\frac{\left(Z_{x z}^{s}\right)^{2} h\left[v_{x}^{s}\right]_{b}^{s}}{\left(Z_{x x}^{s}+Z_{z z}^{s}\right)^{2}}\right. \\
& \left.+\varepsilon \frac{Z_{x z}^{s} Z_{z z}^{s} h\left[v_{x}^{s}\right]_{b}^{s}}{\left(Z_{x x}^{s}+Z_{z z}^{s}\right)^{2}}+\varepsilon f_{D} a_{z}^{s}\left|v_{x}^{s}\right| \frac{2 Z_{x z}^{s} h}{Z_{x x}^{s}+Z_{z z}^{s}}\right)
\end{aligned}
$$

where $a_{z}^{s}=\sqrt{\frac{8}{27} \sin \left(\phi_{b e d}\right)}$ and $\phi_{b e d}$ is the bed friction angle. We omitted $\varepsilon^{2}$-terms and worked with the assumption of $\int_{b}^{s} Z_{i j}^{s} \frac{\partial v_{\kappa}}{\partial x} d z=\int_{b}^{s} Z_{i j}^{s} d z \int_{b}^{s} \frac{\partial v_{\kappa}}{\partial x} d z$. Here, for the sake of simplicity, $Z_{i j}^{s}$ is restricted to one balance equation in the $x z$-plane, assuming isotropic intergranular stress with $Z_{x x}^{s}=Z_{z z}^{s}$ and applying the steady-state correlation $Z_{x z}^{s}=-(3 / \sqrt{2}) \mathrm{a}_{z}^{s} Z_{x x}^{s}$. Thus, the 6 components of the full symmetric 3D tensorial variable are reduced to one component, accounting for the $1 \mathrm{D}$-state. Also note that we set $f_{s}=f_{D}=1$. A range of dimensionless numbers,

$$
\mathrm{Ga}=\frac{\rho g L^{3}}{\mu_{0}}, E u=\frac{C_{e}^{f} \rho^{f} g H}{\rho g L}=\frac{\Delta \varpi}{\rho v^{2}}, \mathrm{~N}_{z}=\frac{\rho \delta z\left(Z_{x x}^{0, s}+Z_{z z}^{0, s}\right)}{\rho g L}
$$

is introduced into the non-dimensional stress tensor, describing the influence of different terms. The relation of gravitational forces to inner friction is depicted by the Galilei number, $\mathrm{Ga}$. It appears with the viscosity terms. For the dimensionless intergranular friction, we introduce a number $\mathrm{N}_{\mathrm{z}}$ in conjunction with the hypoplastic frictional stress terms. It is assembled 
with the proportion of hypoplastic forces to inertial forces. Furthermore, the Euler number Eu gives the influence of a partial pressure $C_{e}^{f} \rho^{f} g H \sim \Delta \varpi$ in relation to inertial forces $\rho g L \sim \rho v^{2}$. It specifies the influence of the pore-fluid extra pressure and appears in conjunction with the non-dimensional extra pore-fluid pressure in the following.

\section{NUMERICAL SIMULATION}

Below, the partial differential system assembled is numerically solved. The numerical instabilities apparent in the simulations of these kinds of systems have already been discussed by Ref. [18] and call for a sophisticated scheme. Subsequently, different schemes for systems with large spatial gradients and dominant convection terms have been compared in Ref. [19]. According to their results, a high-resolution scheme with total variation diminishing property is chosen. We deploy a central-upwind (CU) scheme, developed in the works of Kurganov et al., see Refs. [20, 21], and further deployed with special focus on depth-integrated Saint-Venant systems and Savage-Hutter type models in Ref. [22]. These robust schemes are Godunov-type finite volume methods, capable of solving systems of balance laws by specially treating source terms, non-conservative products and flux terms in conjunction with local propagation speeds. As a core property, the scheme is both shock-capturing and non-oscillatory due to the satisfaction of the total-variation diminishing property. As for discretization in time, a classical two-step Runge-Kutta scheme is applied. For the explicit time stepping, the CFL condition gives the time step size. For details on the derivation of the scheme, its properties and limitations, please see Ref. [22]. The Nessyahu-Tadmor (NT) scheme, a forerunner of these schemes, has been proposed by Ref. [23]. Please note that, for the sake of brevity, we do not give details on the discretization here.

To solve the system, the balance equations are rewritten in vectorial form,

$$
\partial t(\mathrm{U})+\partial_{x}(\mathrm{~F}(\mathrm{U}))=\mathrm{S}(\mathrm{U})+\mathrm{C}_{1}(\mathrm{U}) \partial_{\mathrm{x}}\left(\mathrm{C}_{2}(\mathrm{U})\right)
$$

with the vectors of unknowns in terms of conservative variables

$$
U_{s}=\left(\begin{array}{l}
h v^{s} \\
h v^{s} v_{x}^{s} \\
h Z_{x z}^{s}
\end{array}\right), U_{f}=\left(\begin{array}{l}
h v^{f} \\
h v^{f} v_{x}^{f} \\
h \varpi_{e}^{f}
\end{array}\right)
$$

the down-slope flux $\mathbf{F}(\mathbf{U})$, a source-term $\mathbf{S}(\mathbf{U})$ and the non-conservative term $\mathrm{C}_{1}(\mathrm{U}) \partial_{\mathrm{x}}\left(\mathrm{C}_{2}(\mathrm{U})\right)$. In contrast to the NT-scheme, the flux is propagated with local speeds of propagation, which

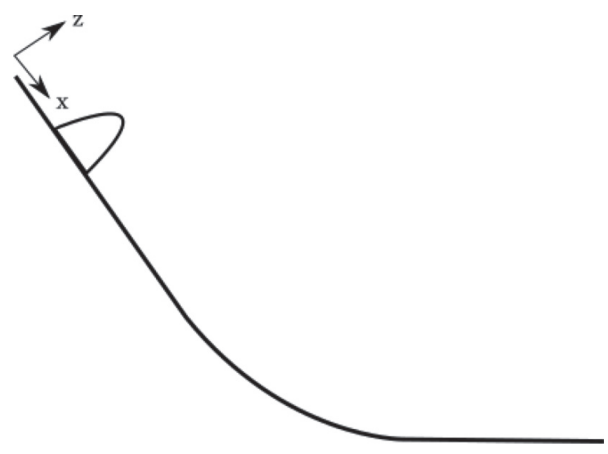

Figure 1: Debris flow problem geometry: initial pile on a slope. 
leads to less numerical dissipation. Furthermore, no staggered grid is needed. For the treatment of the non-conservative products in the form of $\phi_{1} \partial_{x} \phi_{2}$, described in Ref. [22], numerical derivatives are constructed with non-linear limiters. Please note that desingularization, as it is described in Ref. [22], is not considered here.

Below, we present the results of different numerical studies. First, we apply numerical simulations for the case of a simple inclined plane without bed curvature or any complex terrain, which runs out into the horizontal plane. The transition between ramp and horizontal plane is smooth. The pile is released out of rest at time $t=0$, with a parabolic initial geometry, given as $h(t=0, x)=h_{0}\left(1-\left(x-x_{0}\right)^{2} / 16\right)$, with $h_{0}=1, x_{0}=5$. The computational domain is $x \in$ $[-10,80]$, discretized by a number of grids, $n_{x}=500$. The basic parameters are given in the appendix. The inclination angle is given as

$$
\vartheta(x)= \begin{cases}40^{\circ} & 0 \leq x \leq 24 \\ 40^{\circ}(1-(x-24) / 10 & 24<x<34 \\ 0^{\circ} & x \geq 34 .\end{cases}
$$

\subsection{Development of height}

The height profile for the basic system, i.e. without the influence of the both extra pore-fluid pressure and hypoplastic stress, is displayed in Fig. 2. Released from rest and driven by gravity, the pile accelerates and elongates, as the front moves faster than the tail. In the runout, the front decelerates and piles up. Here, we reproduce the results of Ref. [24], since the different forms of flux-discretization between their NT-scheme and the Central Upwind scheme do not considerably affect the results. Please note that the resulting height profile in Ref. [24] is shown for a $2 \mathrm{D}$ flow with an ellipsoidal shell, so the pile also propagates in the cross-slope direction. The similarity of the results shows that, for this particular case, the additional buoyant term plays no major role. The apparent height profile is considered as a referential basis in order to compare results in the following.

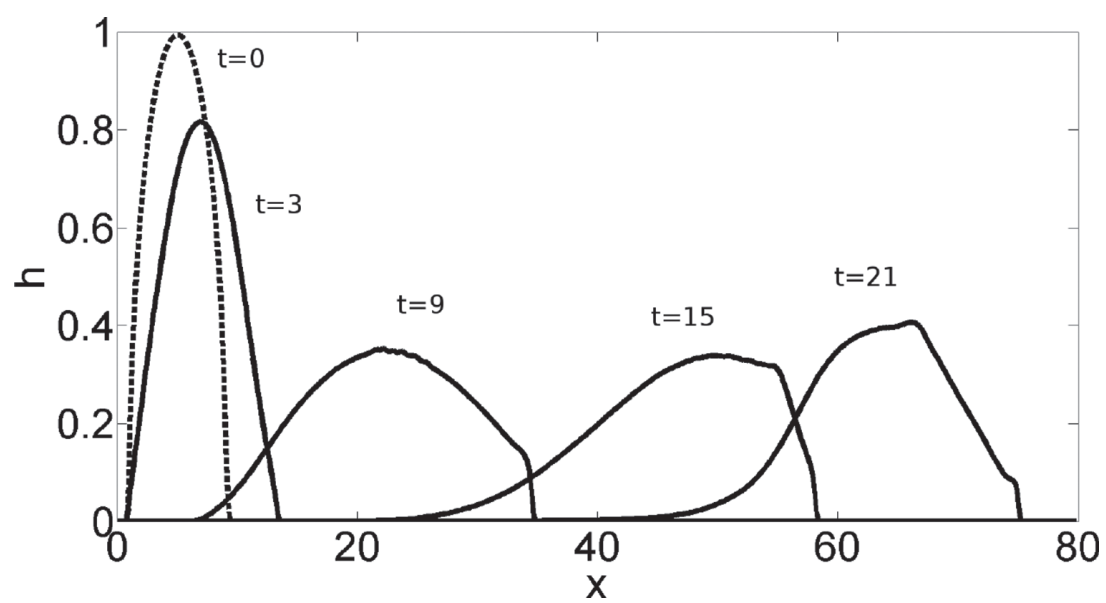

Figure 2: Height-profile development for times $t=0$ (dashed line), $t=3, t=9, t=15$ and $t=21$, with $N_{\mathrm{z}}=0, E u=0$. 


\subsection{Influence of extra pore-fluid pressure}

We now impose the influence of the pore-fluid extra pressure via the variation of the dimensional number and also of the initial value $\varpi_{e}^{0}$. While the consideration of a dynamical extra pore-fluid pressure in the development of the velocities is adjusted through changes in $\mathrm{Eu}$, an increased initial pressure represents the destabilization of soil after heavy rainfall, see Ref. [9]. As it can be seen in Fig. 3, the mobility of the flow is increased due to extra porefluid pressure. This results of the reduced solid bed friction and the influence of the extra pressure on the driving pressure gradient. It is apparent that the influence of the extra pressure is most visible at a later time. This implies firstly, that the extra pressure develops with the flow dynamics and secondly, that the effect of extra pressure is to prolongate the movement of the debris flow after it has accelerated. Both observations match expectations.

Next, the influence of an increased initial pore fluid pressure is investigated. In nature, this kind of triggering mechanism can condition the onset of the avalanches. Here, as the debris flow accelerates due to gravity, an immediately increased mobility can be observed, i.e. the initial level of the extra pore fluid pressure significantly influences the acceleration, as it can be seen in Fig. 4 and in contrast to Fig. 3.

\subsection{Influence of Hypoplastic stress}

The dimensionless number $N_{\mathrm{z}}$ is now set to a non-zero value. Due to the introduction of an intergranular stress, the debris piles up with a steeper front and a visible peak, as it is known from piles of granular material. It appears that the dynamics of the flow are influenced rather little, but interesting variation can be observed at the areas in which the bulk piles up again after being slowed down, as can be seen in Fig. 5 .

In order to further investigate the influence of the hypoplastic stress-term in the solid $\mathrm{x}$ momentum balance, another case is considered. In a horizontal plane, i.e. $\vartheta(x)=0$, a pile with flat heading, dissolving out of rest is investigated. This corresponds to the deposition of granular material. In doing so, the structure-preserving influence of hypoplasticity is

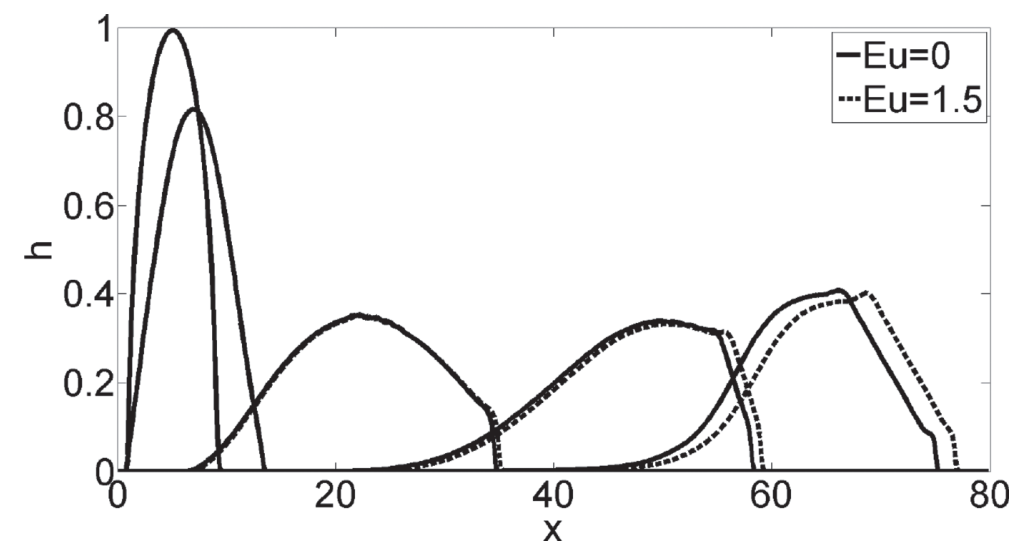

Figure 3: Height-profile development for times $t=0, t=3, t=9, t=15$ and $t=21$, with $N_{\mathrm{z}}=0$ and varying influence of the extra pore-fluid pressure, i.e. changing $E u$. The initial extra pore-fluid pressure is considered as $\varpi_{e}^{0}=0$. The results with $E u=1.5$ are plotted with dashed lines, the solid lines are for $E u=0$. 


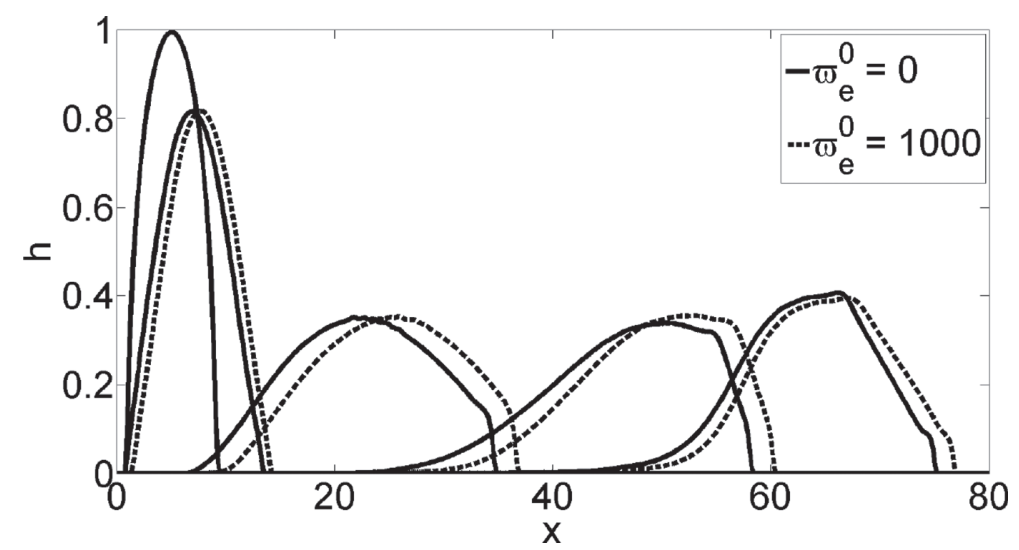

Figure 4: Height-profile development for times $t=0, t=3, t=9, t=15$ and $t=21$, with $N_{\mathrm{z}}=0$, $E u=1.5$ and changing initial extra pore-fluid pressure $\varpi_{e}^{0}$.

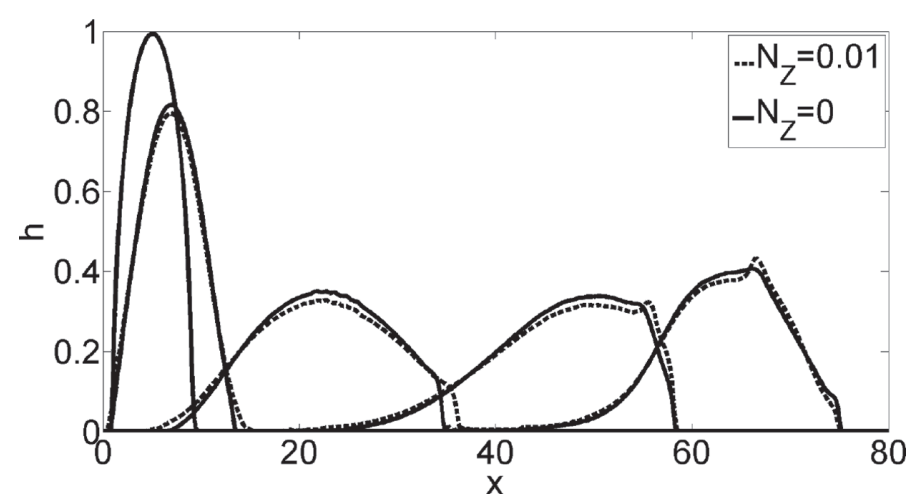

Figure 5: Height-profile development for times $t=0, t=3, t=9, t=15$, and $t=21$, with $E u=0$ and changed $N_{z}$ (dashed line). Initial hypoplastic stress $Z_{x z}^{s 0}=100$.

examined, since this this flow regime seems to be most likely affected by the intergranular stress. Figure 6 displays the influence of hypoplastic internal friction on maintaining a steep front, i.e. preventing the material from dissolving fluid-likely due to a resisting intergranular contact stress.

\subsection{Discussion}

In a first attempt to investigate on the influence of an extra pore-fluid pressure and an intergranular contact stress, implemented in terms of hypoplasticity, the results match expectation. The extra pore-fluid pressure increases the mobility of the mixture, as it reduces the friction and increases the driving pressure. The implementation via an own evolution equation enables the system to consider the temporal development due to the development of the soil structure, i.e. dilatancy. Furthermore, via the initial conditions, the extra porefluid pressure can serve an initially driving mechanism as well. Moreover, the intergranular contact friction shows its influence on the height profile for the process of deposition and processes close to 


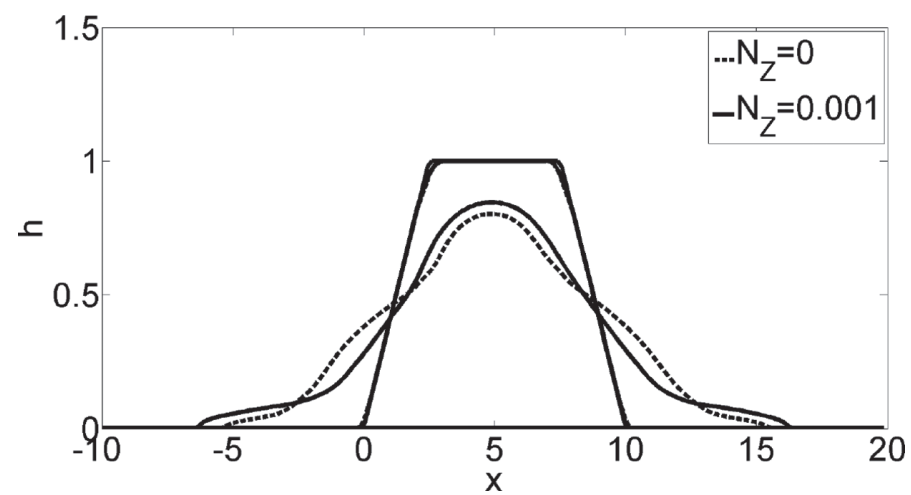

Figure 6: Height-profile development for times $t=0, t=0.5$ and $t=31.3$, with $E u=0$ and changing $N_{\mathrm{z}}$.

equilibrium. In this range with rather small velocities, the intergranular friction prevents the granular body from dissolving in a fluid-like way.

First investigations show the potential of the comprehensive consideration of these specific physical mechanisms. Nonetheless, further work both in modeling the interdependency and in numerical investigations are considered fruitful to fully understand the impact of the new modeling approach. Since, to the best of the authors' knowledge, the combined integration of both hypoplasticity and extra pore-pressure evolution has not been carried out in previous works, the modeling of the mutual interferences is still in an early stage. An important link is granular dilatancy and, in general, the deformational behavior of granular materials. The apparent rate-dependency of granular material manifests in hysteresis-like de-formational behavior. Dilatancy describes the relation between shear and volume changing, which is dependent on the intergranular forces. In return, these intergranular contact forces are themselves depending on the pore pressure. Like this, the modeling of the dilatancy part, $\tan (\phi)$, in the extra pore-fluid pressure source term could be connected to the hypoplastic stress tensorial variables $Z_{i j}^{s}$ and the implementation of the hypoplastic intergranular friction in the solid-stress could be linked to pore-fluid pressure.

Consideration of the source term in the balance equation for the development of the solid volume fraction $n^{\alpha}$ could account for the specific peculiarity of the microstructures of granular materials in their development, and account for e.g. dilatancy, as it is done by Ref. [9]. Also of importance is the investigation on the possible cases in which the additional buoyant term gains significant influence.

Beside these modeling aspects, further investigation of 3D flow with a system of depth integrated equations and a full set of hypoplastic equations will be considered in future works as well as comparison with experimental findings. Also here, detailed insight in the stressstrain relations and the modeling of distinct coefficient will take place.

\section{CONCLUSION}

In this work, we propose a debris flow model that accounts for the evolution of both an extra porefluid pressure and intergranular friction. Based on the results of the exploitation of the entropy principle in its formulation by Müller and Liu, we non-dimensionalize and depth-integrate the balance equations. Comparison shows that the model is capable of reproducing previous modeling attempts but enhances the basic framework of momentum balances with additional terms, 
accounting for distinct physical mechanisms. The extra pore-fluid pressure enhances mobility and reduces the apparent friction, whereas the hypoplastic stress incorporates the anelastic deformational behavior of granular flows. Numerical simulation proves the meaningfulness of these additional quantities. The dynamic pore pressure increases the mobility of the flow, while the intergranular friction counteracts fluid-like dissolving and gains importance during the deposition.

In subsequent works, the model will be tested for 3D cases and compared to findings with other models and experimental data. Extended parametric studies will be applied.

\section{ACKNOWLEDGEMENTS}

We would like to express our gratitude for the financial support of the Deutsche Forschungsgemeinschaft (DFG) for this work within the project number WA 2610/3-1.

\section{REFERENCES}

[1] Kolymbas, D., A rate-dependent constitutive equation for soils. Mechanics Research Communications, 4(6), pp. 367-372, 1977. https://doi.org/10.1016/0093-6413(77)90056-8

[2] Kolymbas, D., A generalized hypoelastic constitutive law. In Proceeding of the XI International Conference on Soil Mechanics and Foundation Engineering, San Francisco, volume 5, page 2626, 1985.

[3] Kolymbas, D., An outline of hypoplasticity. Archive of Applied Mechanics, 61(3), pp. 143-151, 1991.

[4] von Terzaghi, K., Die Berechnung der Durchlässigkeitsziffer des Tones aus dem Verlauf der hydrodynamischen Spannungserscheinungen. Sitzungsberichte der Akademie der Wissenschaften in Wien, Mathematisch-Naturwissenschaftliche Klasse, Abteilung IIa, 123, pp. 125-138, 1923.

[5] von Terzaghi, K., The shearing resistance of saturated soils and the angle between the planes of shear. Proceedings of the 1st International Conference on Soil Mechanics and Foundation Engineering, 1, pp. 54-56, 1936.

[6] Iverson, R.M., The physics of debris flow. Reviews of Geophysics, 35(3), pp. 245-296, 1997. https://doi.org/10.1029/97rg00426

[7] Savage, S.B. \& Iverson, R.M., Surge dynamics coupled to pore-pressure evolution in debris flows. Millpress, Vol. 1, pp. 503-514, 2003.

[8] George, D.L. \& Iverson, R.M., A two-phase debris-flow model that includes coupled evolution of volume fractions, granular dilatancy, and pore-fluid pressure. Italian Journal of Engineering Geology and Environment, 10, pp. 2011-03, 2011.

[9] Iverson, R.M. \& George, D.L., A depth-averaged debris-flow model that includes the effects of evolving dilatancy. I. Physical basis. In Proceedings of the Royal Society A: Mathematical, Physical and Engineering Sciences, volume 470. The Royal Society, 2014.

[10] Müller, I. \& Liu, I-S., Thermodynamics of mixtures of fluids. In C. Truesdell (Ed.), Rational Thermodynamics. Springer, 1984.

[11] Müller, I., A thermodynamic theory of mixtures of fluids. Archive for Rational Mechanics and Analysis, 28(1), pp. 1-39, 1968.

https://doi.org/10.1007/bf00281561 
[12] Liu, I-S., Method of Lagrange multipliers for exploitation of the entropy principle. Archive for Rational Mechanics and Analysis, 46(2), pp. 131-148, 1972. https://doi.org/10.1007/bf00250688

[13] Heß, J., Wang, Y. \& Hutter, K., Thermodynamically consistent modeling of granularfluid mixtures incorporating pore pressure evolution and hypoplastic behavior. Continuum Mechanics and Thermodynamics, 29(1), pp. 311-343, 2017. https://doi.org/10.1007/s00161-016-0535-9

[14] Schneider, L. \& Hutter, K., Solid-fluid mixtures of frictional materials in geophysical and geotechnical context. Advances in Geophysical and Environmental Mechanics and Mathematics. Springer, 2009.

[15] de Boer, R. \& Ehlers, W., The development of the concept of effective stresses. Acta Mechanica, 83(1), pp. 77-92, 1990. https://doi.org/10.1007/bf01174734

[16] Liu, I-S., A solid-fluid mixture theory of porous media. International Journal of Engineering Science, 84, pp. 133-146, 2014.

https://doi.org/10.1016/j.ijengsci.2014.07.002

[17] Pudasaini, S.P., A general two-phase debris flow model. Journal of Geophysical Research, 117, pp. 1-28, 2012. https://doi.org/10.1029/2011jf002186

[18] Savage, B. \& Hutter, K., The motion of a finite mass of granular material down a rough incline. Journal of Fluid Mechanics, 199, pp. 177-215, 1989. https://doi.org/10.1017/s0022112089000340

[19] Wang, Y. \& Hutter, K., Comparisons of numerical methods with respect to convectively dominated problems. International Journal for Numerical Methods in Fluids, 37(6), pp. 721-745, 2001. https://doi.org/10.1002/fld.197

[20] Kurganov, A. \& Tadmor, E., New high-resolution central schemes for nonlinear conservation laws and convection-diffusion equations. Journal of Computational Physics, 160(1), pp. 241-282, 2000.

https://doi.org/10.1006/jcph.2000.6459

[21] Kurganov, A., Noelle, S. \& Petrova, G., Semidiscrete central-upwind schemes for hyperbolic conservation laws and Hamilton-Jacobi equations. SIAM Journal on Scientific Computing, 23(3), pp. 707-740, 2001.

https://doi.org/10.1137/s1064827500373413

[22] Kurganov, A. \& Miller, J., Central-upwind scheme for savage-Hutter type model of submarine landslides and generated tsunami waves. Computational Methods in Applied Mathematics, 14(2), pp. 177-201, 2014. https://doi.org/10.1515/cmam-2014-0003

[23] Nessyahu, H. \& Tadmor, E., Non-oscillatory central differencing for hyperbolic conservation laws. Journal of Computational Physics, 87(2), pp. 408-463, 1990. https://doi.org/10.1016/0021-9991(90)90260-8

[24] Meng, X. \& Wang, Y., Modelling and numerical simulation of two-phase debris flows. Acta Geotechnica, 11(5), pp. 1027-1045, 2016.

https://doi.org/10.1007/s11440-015-0418-4 
APPENDIX

\begin{tabular}{|c|c|c|c|}
\hline Parameter & Case 1 & Case 2 & Description \\
\hline$\rho^{s}$ & $2500 \mathrm{~kg} / \mathrm{m}^{3}$ & $2500 \mathrm{~kg} / \mathrm{m}^{3}$ & Solid density \\
\hline$\rho^{s}$ & $1000 \mathrm{~kg} / \mathrm{m}^{3}$ & $1000 \mathrm{~kg} / \mathrm{m}^{3}$ & Fluid density \\
\hline$g$ & $9.81 \mathrm{~m} / \mathrm{s}^{2}$ & $9.81 \mathrm{~m} / \mathrm{s}^{2}$ & Gravitational acceleration \\
\hline$\vartheta_{0}$ & $\vartheta_{0}$ & $0^{\circ}$ & Inclination angle \\
\hline$\phi_{b e d}$ & $33^{\circ}$ & $33^{\circ}$ & Bed friction angle \\
\hline$\alpha^{f}$ & 20 & 20 & Navier fluid friction coefficient \\
\hline$c_{D}^{s}$ & 6 & 6 & Drag Coefficient \\
\hline$\kappa_{0}$ & $7.5 \cdot 10^{-9}$ & - & Hydraulic permeability coefficient \\
\hline$a_{D}$ & 1715 & - & Compressibility factor \\
\hline$\sigma_{0}$ & $100 \mathrm{~kg} / \mathrm{m} \mathrm{s}^{2}$ & - & Intergranular basic stress \\
\hline$\delta_{p}$ & $0.001 \mathrm{~m}$ & - & Average particle size \\
\hline$v_{c}^{s}$ & 0.64 & - & Critical volume fraction \\
\hline$v_{\max }^{s}$ & 0.75 & - & Maxmimum volume fraction solid \\
\hline$\kappa_{\varpi 1}$ & 1.1 & - & Dilatancy Coefficient 1 \\
\hline$\kappa_{\varpi 2}$ & 3.3 & - & Dilatancy Coefficient 2 \\
\hline$\mu^{s}$ & $0.54 \mathrm{~kg} \mathrm{~m}^{-1} \mathrm{~s}$ & - & Dynamical viscosity \\
\hline$v_{0}^{s}$ & 0.5 & 0.5 & Initial volume fraction solid \\
\hline$v_{0}^{f}$ & 0.5 & 0.5 & Initial volume fraction fluid \\
\hline$v_{0}^{x}$ & 0 & 0 & Initial velocity \\
\hline$Z_{x z}^{s 0}$ & $100 \mathrm{~kg} / \mathrm{ms}^{2}$ & $100 \mathrm{~kg} / \mathrm{ms}^{2}$ & Initial intergranular stress \\
\hline$\varpi_{e}^{0}$ & $0-1000 \mathrm{~kg} / \mathrm{ms}^{2}$ & - & Initial extra pressure \\
\hline$\in$ & 1 & 1 & Height versus length ratio \\
\hline$f_{s^{\prime}} f_{D}$ & 1 & 1 & Hypoplastic coefficients \\
\hline
\end{tabular}

